\title{
IEEE Dynamic Spectrum Access Networks (DYSPAN) Standards Committee
}

\author{
Hiroshi Harada, Yohannes D. Alemseged \\ NICT \\ Yokosuka, Japan \\ E-mail:\{harada,yohannes\}@nict.go.jp
}

\author{
Oliver Holland \\ King's College of London \\ London, UK \\ E-mail: oliver.holland@kcl.ac.uk
}

\begin{abstract}
This article presents the latest status and achievements of the IEEE Communication Society (ComSoc) Standards for Dynamic Spectrum Access Networks (DYSPAN), formerly IEEE Standards Coordinating Committee 41, that develops standards in the areas of dynamic spectrum access, cognitive radio, interference management, coordination of wireless systems, advanced spectrum management, and policy languages for next generation radio systems under the sponsorship of the IEEE communications society. The article also envisions future directions for the DYSPAN Committee. This paper represents the personal opinion of the authors, not the official opinion of the IEEE ComSoc DYSPAN standards committee.
\end{abstract}

Keywords-component; IEEE ComSoc DYSPAN standards commitee, dynamic spectrum access network; cognitive radio,

\section{INTRODUCTION}

The evolution of modern wireless communications and the upgrading of existing systems to cater for higher throughput and higher mobility are challenged by radio scarcity in allocated spectrum bands. Nevertheless, recent studies reveal that various spectrum bands which are allocated through fixed spectrum assignment to alternative usage purposes are underutilized [1]. One way to alleviate problems caused by the lack of available allocated spectrum for pioneering devices and systems would therefore be for those devices and systems to be able to dynamically access alternative locally/temporally unused spectrum bands. Of course, this process must not cause any disruption to the incumbent users of those alternative bands. For instance regulatory bodies such as The United States Federal Communication Commission (FCC) have released a series of Memorandum of Opinion and Order in the matter of unlicensed operation in TV broadcast bands that addresses on allowing temporarily unused television bands without causing harmful interference to the TV broadcasts.

The International Telecommunication Union (ITU) defines cognitive radio system (CRS) as"A radio system employing technology that allows the system: to obtain knowledge of its operational and geographical environment, established policies and its internal state; to dynamically and autonomously adjust its operational parameters and protocols according to its obtained knowledge in order to achieve predefined objectives; and to learn from the results obtained [2]. The CRS can achieve DSA to solve the problem of spectrum scarcity [3]. The two prominent types of CRSs are a network-centric one termed "heterogeneous type" and"spectrum sharing type" [4]. Currently, DYSPAN standards cover both such DSA realms:"heterogeneous type" and "spectrum sharing type".

In the heterogeneous type CRS, the "primary" or "legacy" system(s) with rights to access the spectrum band(s) negotiate or manage allocations dynamically among themselves, usually through a centralized server or collaboration of servers. One or several operators operate several radio access networks (RANs) using same or different RATs. Frequency bands allocated to these RANs are fixed. There are different types of base stations and terminals. One is legacy type designed to use a particular radio access technology (RAT) to provide wireless connection between base stations and terminals. Another is reconfigurable type having capability to reconfigure itself to use different frequency bands allocated to the operator and to use different RATs as specified by the radio regulations for these frequency allocations. In the spectrum sharing type CRS, on the other hands, secondary spectrum access (i.e., access of locally/temporally unused spectrum bands by a system that doesn't own the conventional"rights" to that spectrum) is done by devices without (or with very limited) communication with the primary system(s). Several RANs using same or different RATs can share the common frequency band. Both types of CRSs need to have sensing mechanism in the radio equipment.

Recently, research and development centres and industries have been investigating the functional architectures and interfaces of heterogeneous type and spectrum sharing type, as well as appropriate algorithms, overall network performance, network complexity, technical maturity, etc. The outcomes of this extensive research and development have indicated that there is a huge market potential for DSA to be utilized in the wireless communications world. The success of this market depends not only on technological aspects but also on the availability of supporting international standards. This is important, as to support both heterogeneous type CRS and spectrum sharing CRS, different types of systems must directly and indirectly interact, and legacy systems must be appropriately protected from any potential radio-interference or other negative effects due to CRS.

In response to this need, the IEEE Communication Society (ComSoc) and the IEEE Electromagnetic Compatibility Society (EMC Society) collaborated in early 2005 to establish the IEEE P1900 Standards Committee, the objective of which was to develop supporting standards dealing with new technologies and techniques for next generation radio and advanced spectrum management [5]. On March 22, 2007, the 
IEEE Standards Board approved the reorganization of the IEEE 1900 effort into Standards Coordinating Committee 41 (SCC41)“Dynamic Spectrum Access Networks". Since the formation of IEEE SCC41, industries, research institutes, and organizations from around world, especially from Asia, Europe, and North America, have been actively contributing to the development of a series of standards in the area of DSA. The concerted effort has produced the first standard under SCC41 which was published in 2008. As of December 16, 2010, the SCC41 standards development activities and project responsibilities have been transferred to the IEEE ComSoc standards board (CSSB) where the 1900.x projects run under a common umbrella of DYSPAN standards committee (DYSPAN SC).

\section{SCOPE AND STRUCTURE}

IEEE DYSPAN SC develops standards related to "Dynamic Spectrum Access Networks". Its scope includes interference management, coordination of wireless technologies, network management, and information sharing to support new techniques and methods of DSA, with a focus on improved use of spectrum. Currently five WGs are actively engaged in developing draft standards. In general there are two types of WG membership: individual-based and entitybased. The IEEE 1900.1, 1900.2, 1900.5 and 1900.6 WGs are individual-based, and the IEEE 1900.4 WG is entity-based. DYSPAN SC members are affiliated with research institutes, manufacturers, telecommunications operators, universities, and various other interests groups from American, European and Asian countries.

\section{A. IEEE 1900.1 WG}

The scope of this WG is to provide technically-precise definitions and explanations of the key concepts of DSA and related technologies, in order to facilitate the development of these technologies by clarifying terminologies and how these technologies and the ways in which these technologies relate to each other. Terminologies are classified into four categories.

Advanced radio system concepts: this category includes 9 items, such as adaptive radio, cognitive radio, reconfigurable radio, software-defined radio, etc. As an example, the definition of term "cognitive radio" is "a) A type of radio in which communication systems are aware of their environment and internal state and can make decisions about their radio operating behaviour based on that information and predefined objectives. b) Cognitive radio [as defined in item a)] that uses software-defined radio, adaptive radio, and other technologies to adjust automatically its behaviour or operations to achieve desired objectives."

Radio system functional capabilities: this category contains ten items, such as cognitive control mechanism, adaptive modulation, cognitive control mechanism, etc. As an example, the definition of term "adaptive modulation" is "A radio system function for adjusting the modulation format."

Network technologies: this category contains 4 items as follows: cognitive radio network, composite network, reconfigurable networks, and dynamic spectrum access network. As an example, the definition of term "reconfigurable networks" is "Networks that have a capability to be reconfigured dynamically (e.g., change in network topology) or a capability to change communication protocols dynamically under the control of network management services."

Spectrum management: this category includes 58 items, such as coexistence mechanism, collaborative spectrum usage, spectrum sharing, DSA, etc. As an example, the definition of term "Dynamic Spectrum Access" is "The real-time adjustment of spectrum utilization in response to changing circumstances and objectives.”

The IEEE 1900.1 standard was published in September 2008 with title "IEEE Standard Definitions and Concepts for Dynamic Spectrum Access: Terminology Relating to Emerging Wireless Networks, System Functionality, and Spectrum Management." [6].

\section{B. IEEE 1900.2 Standard}

This WG provides technical guidelines for analyzing the potential for coexistence and interference between radio systems operating in the same frequency band or between different frequency bands. The IEEE 1900.2 standard was published in July 2008, with title "IEEE Recommended Practice for the Analysis of In-Band and Adjacent Band Interference and Coexistence between Radio Systems" [7]. The standard document provides a structure and describes a method for analyzing the interference between radio services under a variety of coexistence scenarios, as well as the details about variables to be used for analysis. The standard document also provides a framework to help the understanding of assumptions, dependencies, and relative importance of the variables of the problem.

\section{IEEE $1900.4 \mathrm{WG}$}

The 1900.4 WG has developed the IEEE standard 1900.4 for "Architectural Building Blocks Enabling Network-Device Distributed Decision Making for Optimized Radio Resource Usage in Heterogeneous Wireless Access Networks" [8]. This is the world-first standard related to configuration network aspects of CRS. Currently, two ensuing projects are active within the 1900.4 WG. The first project is P1900.4.1 "Interfaces and Protocols Enabling Distributed Decision Making for Optimized Radio Resource Usage in Heterogeneous Wireless Networks." The second project is P1900.4a "Architecture and Interfaces for Dynamic Spectrum Access Networks in White Space Frequency Bands."

IEEE standard 1900.4 is a baseline standard. Its main goal is to provide a framework for developing intelligent management system having the capability to optimize spectrum usage across different frequency bands, RATs, and operators. To reach this goal, the standard defines the architecture of the management system, which is comprised of the component entities of the management system, SAPs of these entities, and interfaces between them. IEEE standard 1900.4 considers three use cases shown in Figure 1. These use cases are called distributed radio resource usage optimization, dynamic spectrum assignment, and dynamic spectrum sharing. 
In the distributed radio resource usage optimization use case, one or several operators operate several radio access networks (RANs) using same or different RATs. Frequency bands assigned to these RANs are fixed. Also, reconfiguration of radio equipment on network side is not considered. These RANs provide services to their users, which use different terminals. One type of terminals is legacy terminals, designed to use a particular RAT. Such terminals can connect to one particular operator or to other operators having roaming agreements with the home operator. Another type of terminals is reconfigurable terminals. Such terminals have capability to reconfigure themselves to use different RATs. Correspondingly, such terminals can handover between different RANs using different RATs and operated by different operators.

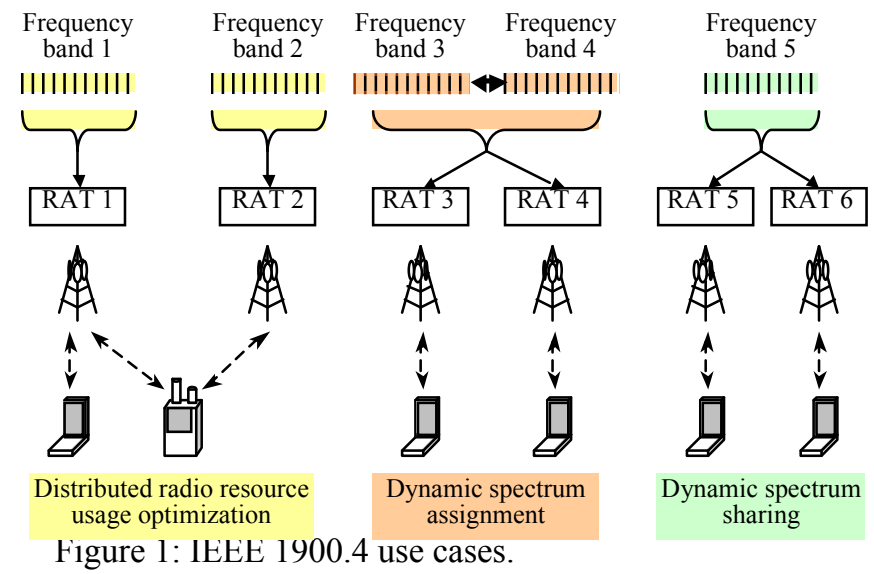

In dynamic spectrum assignment use case, one or several operators operate several RANs using same or different RATs in different frequency bands. To improve radio resource usage, configuration of these frequency bands can be dynamically changed. One example application of this use case is joint management of several RANs within one operator, where spectrum allocated to this operator is dynamically redistributed between its RANs. Another example is spectrum trading between different operators.

In dynamic spectrum sharing use case, several RANs using same or different RATs can share the same frequency band. One example of this use case is when several RANs operate in unlicensed spectrum. Another example is when a secondary system operates in white space of a TV broadcast operator frequency band. The architecture defined in the IEEE standard 1900.4 is shown in Figure 2. It defines management entities and interfaces comprising the intelligent management system to be deployed on top of an existing wireless environment.

Four management entities are defined on the network side: the Operator Spectrum Manager (OSM), the RAN Measurement Collector (RMC), the Network Reconfiguration Manager (NRM) and the RAN Reconfiguration Controller (RRC).

The Operator Spectrum Manager is the entity that enables operator to control dynamic spectrum assignment decisions to be made by the NRM. The RAN Measurement Collector is the entity that collects RAN context information and provides it to the NRM. The Network Reconfiguration Manager is the entity that manages RANs and terminals for network-terminal distributed optimization of radio resource usage and improvement in quality-of-service. The RAN Reconfiguration Controller is the entity that controls reconfiguration of RANs based on requests from the NRM.

To support scalable operation, the RMC, the NRM, and the RRC may be implemented in a distributed manner. Three management entities are defined on the terminal side: the Terminal Measurement Collector (TMC), the Terminal Reconfiguration Manager (TRM) and the Terminal Reconfiguration Controller (TRC). Each terminal has one TMC, one TRM, and one TRC.

The Terminal Measurement Collector is the entity that collects terminal context information and provides it to the TRM.

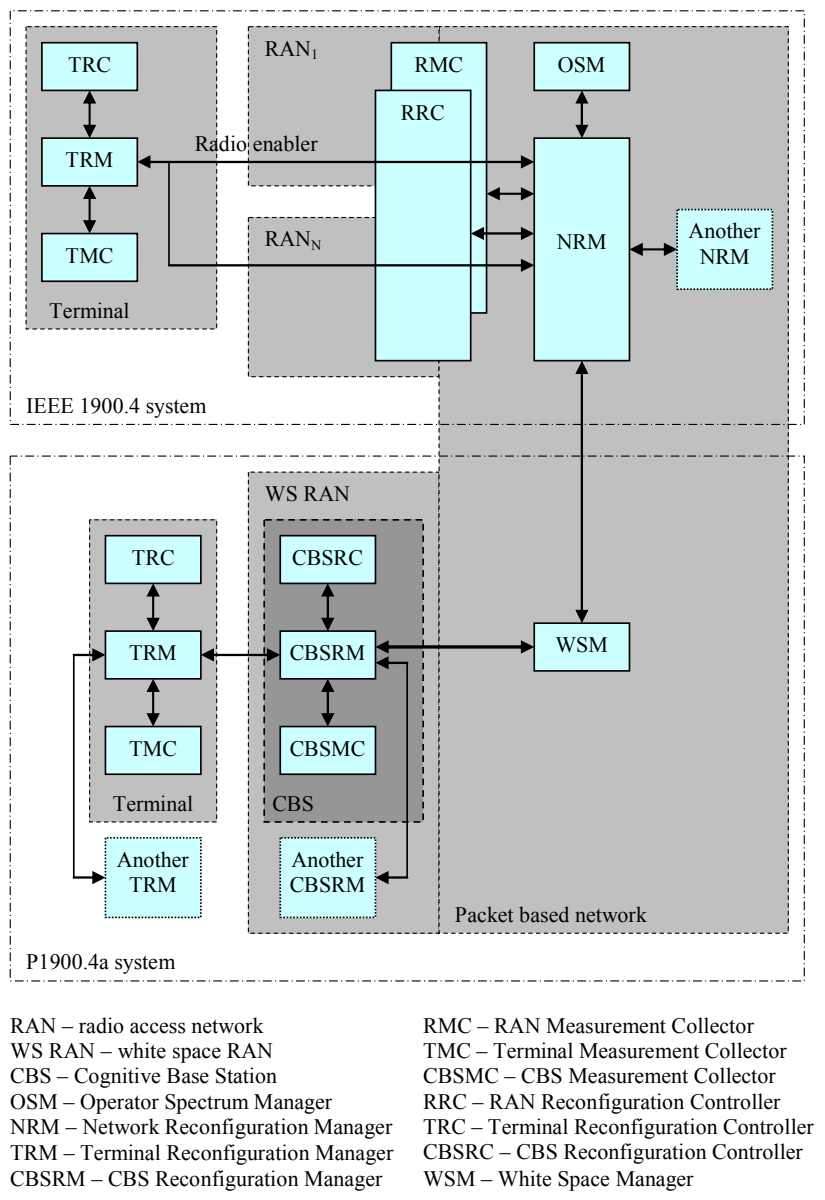

Figure 2: IEEE 1900.4 architecture.

The Terminal Reconfiguration Manager is the entity that manages the terminal for network-terminal distributed optimization of radio resource usage and improvement of quality-of-service. This optimization is performed within the framework defined by the NRM and expressed by radio resource selection policies, and in a manner consistent with user preferences. The Terminal Reconfiguration Controller is the entity that controls the reconfiguration of terminal based on requests from the TRM. 
Development of the draft standard P1900.4.1 "Interfaces and Protocols Enabling Distributed Decision Making for Optimized Radio Resource Usage in Heterogeneous Wireless Networks" started in March 2009.

P1900.4.1 uses the IEEE standard 1900.4 as a baseline standard. The purpose of P1900.4.1 is to provide detailed description of interfaces and service access points defined in the IEEE standard 1900.4 enabling distributed decision making in heterogeneous wireless networks and obtaining context information for this decision making.

Development of the draft standard P1900.4a for "Architecture and interfaces for dynamic spectrum access networks in white space frequency bands" started in March 2009 together with P1900.4.1. It amends the IEEE standard 1900.4 to enable mobile wireless access service in white space frequency bands without any limitation on used radio interface (physical and media access control layers, carrier frequency, etc) by defining additional components of the IEEE 1900.4 system. Currently considered architecture of P1900.4a is shown in Figure 2. Compared to IEEE standard 1900.4, four new entities are currently considered in P1900.4a: the Cognitive Base Station (CBS) Measurement Collector (CBSMC), the CBS Reconfiguration Manager (CBSRM), the CBS Reconfiguration Controller (CBSRC), and the White Space Manager (WSM).

The CBS Measurement Collector is the entity that collects CBS context information and provides it to the CBSRM. Each CBS has one CBSMC. The CBS Reconfiguration Manager is the entity that manages CBS and terminals for networkterminal distributed optimization of spectrum usage. Each CBS has one CBSRM. The CBS Reconfiguration Controller is the entity that controls reconfiguration of CBS based on requests from the CBSRM. Each CBS has one CBSRC. The White Space Manager can be considered as a substitution of the OSM and the NRM in P1900.4a. This entity enables collaboration between P1900.4a system and IEEE 1900.4 system, provides regulatory context information to the CBSRM, and enables communication between the CBSRM and white space database.

\section{IEEE $1900.5 \mathrm{WG}$}

This WG is developing a standard on Policy Language and Policy Architectures for Managing Cognitive Radio for DSA Applications. The purpose of this WG is to define a policy language (or a set of policy languages or dialects) to specify interoperable, vendor-independent control of Cognitive Radio functionality and behavior for DSA resources and services.

\section{E. IEEE 1900.6 WG}

This WG is developing a standard on Spectrum Sensing Interfaces and Data Structures for Dynamic Spectrum Access and other Advanced Radio Communication Systems [9]. The standard will define the information exchange between spectrum sensors and their clients in radio communication systems. The logical interface and supporting data structures used for information exchange are defined abstractly without constraining the sensing technology, client design, or data link between sensor and client.
Figure 3 illustrates the IEEE P1900.6 interface for information exchange, where the client of a spectrum sensor can be Cognitive Engine (CE), Data Archive (DA) or a spectrum sensor $(\mathrm{S})$. The $\mathrm{CE}$ is defined as the portion of the CRS containing the policy based control mechanism and the cognitive control mechanism which must have the knowledge about the current state, a set of attainable states and may have knowledge about cost associated with (state) transitions of the reconfigurable radio platform. And the DA is defined as a logical entity where sensing information obtained from spectrum sensors or other information sources and regulatory and policy information are stored systematically. A sensor is the portion of a radio system that performs spectrum sensing within a CRS. Sensors may also act as clients to other sensors.

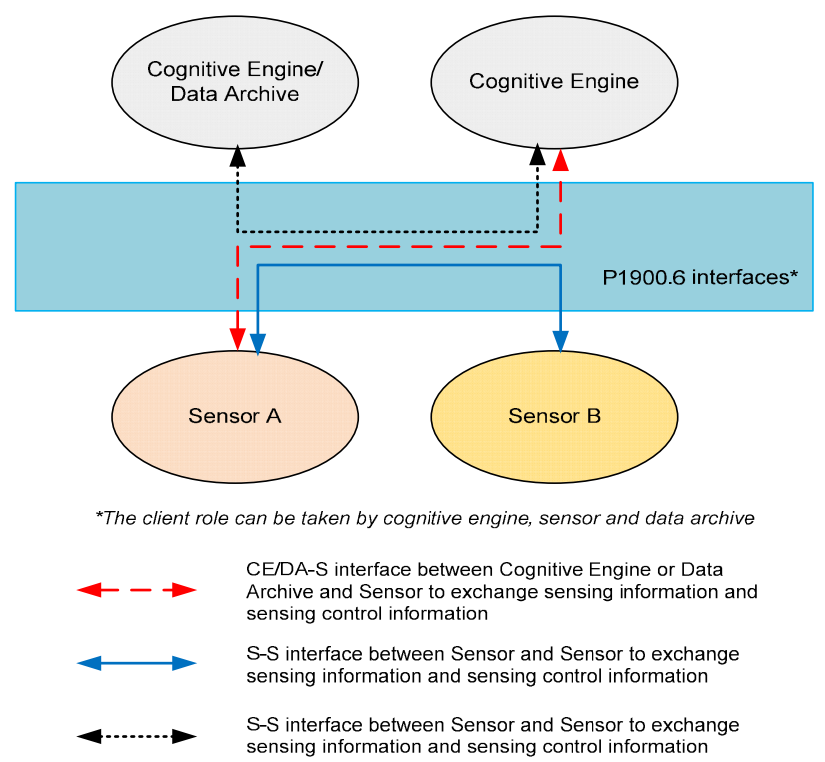

Figure 3: Interface for information exchange between sensors and their clients.
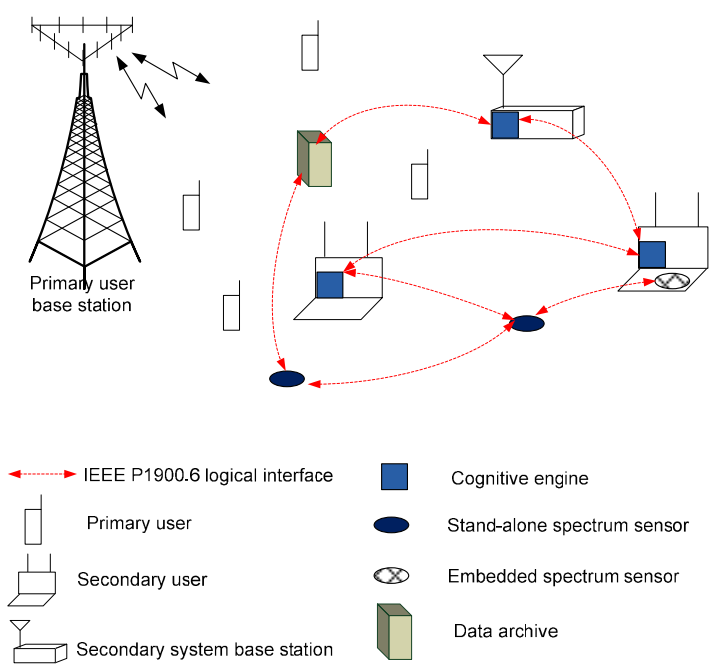

Fig 4. Scenario of CRS where the IEEE P1900.6 interface can be employed. 
The different instantiation of 1900.6 interfaces supports exchanging information either between two logical entities or between a single logical entity and multiple logical entities. In particular the later is used to implement distributed spectrum sensing where multiple sensors collaborate/cooperate to generate quality sensing information. The diagram in Fig. 4 illustrates the interaction of sensors and their clients through the multiple instantiation of the 1900.6 logical interfaces.

The 1900.6 WG defines application SAP, Communication SAP and Measurement SAP in its reference model. The Application SAP (Service Access Point) provides access to the controls and/or applications of the client. The Communication SAP provides media-independent information exchange by providing access to the communication subsystem. The Measurement SAP is used by P1900.6 entities to access P1900.6 compliant services provided by the station's hardware and/or firmware to control the spectrum measurement module such as collocated physical spectrum measurement module, i.e., $\mathrm{ADC} / \mathrm{DAC}$, filtering, signal condition, etc, and acquire measurement data from these modules. The IEEE 1900.6 project was approved as a new standard by the IEEE-SA Standards Board on 2 February 2011.

\section{UPCOMMING PROJECTS AND FUTURE STRATEGY}

\section{A. Upcomming projects}

Currently the DYSPAN standards committee has three new project authorization requests (PARs) on pipeline. The first one is P1900.1a "Amendment to IEEE Standard 1900.1-2008" that deals with addition of new terms and associated definitions to the existing terminology relating to emerging wireless networks, system functionality, and spectrum management amendment. The PAR has been submitted to the IEEE SA NesCom and obtained approval. The second project is P1900.7 "MAC and PHY Specification for fixed, portable, mobile operation of white space dynamic spectrum access radio systems". P1900.7 is intended to focus on the standard specifies radio interface including medium access control (MAC) sublayer and physical (PHY) layer of white space dynamic spectrum access radio systems supporting fixed and mobile operation in frequency bands, such as TV bands, public safety bands, and wireless medical telemetry bands, subject to compliance to national and international radio regulations in these frequency bands. The standard will provide means to support other related IEEE 1900 standards. The corresponding PAR for P1900.7 has also been submitted to IEEE SA NesCom which is still under consideration. The third project is P1900.6a "amendment to IEEE Standard 1900.6 that is anticipated to be published soon". It focuses on procedures, protocols and message format specifications for the exchange of sensing related data, control data and configuration data between spectrum sensors and their clients. In addition, it adds specifications for the exchange of sensing related and other relevant data and specifies related interfaces between the data archive and other data sources.

\section{B. Future Strategy}

As described in this article, the DYSPAN standards committee provides standards on enabling technologies for CRSs that can be leveraged for various other related standardization groups. The results of standards will be contributed to the other standardization bodies as reference and/or baselines. The DYSPAN SC continues to investigate potential areas of DSA and CR through study groups under the committee or individual WGs to initiate new projects that address the imminent need of the wireless communications industry, service providers and users. As noted recently in the FCC NOI released on the $30^{\text {th }}$ of Nov. 20 and published on the $28^{\text {th }}$ of Dec, 2011, in the matter of promoting more efficient use of spectrum through dynamic spectrum use technologies, DYSPAN continues to play a pivotal role in developing standards that facilitate the efficient utilization of the limited spectrum resource.

\section{CONCLUSION}

Motivated by the huge potential market in wireless communications for DSA technologies, and their associated challenges, the DYSPAN standards committee is currently actively developing a series of standards. These standards are intended to support the interoperability of different components and functional blocks of CRSs and facilitate interoperability among DSA systems developed by different manufacturers. This will ensure the reliability of DSA-related processes which is of course highly important to regulators, operators, broadcasters, and other entities. Moreover, these standards will stimulate and facilitate creativity, innovation and entrepreneurship within wireless communications; all essential components as the industry and wider world exit from recession. Moreover, the DSA technologies facilitated by these standards will deliver considerable economic benefit to the wider world through improved communication.

\section{Acknowledgement}

The authors would like to express our cordial thanks to Drs. Stanislav Filin, Ha Nguyen Trans, and Chen Sun for their extraordinary contributions to this paper.

\section{REFERENCES}

[1] G. Staple and K. Werbach, "The end of spectrum scarcity," IEEE Spectrum, vol. 41, no. 3, pp. 48-52, Mar. 2004.

[2] Definitions of Software Defined Radio (SDR) and Cognitive Radio System (CRS), Report ITU-R SM.2152, September 2009.

[3] H. Harada et al., "A Software Defined Cognitive Radio System: Cognitive Wireless Cloud,” IEEE. GLOBECOM 2007, pp. 294-299.

[4] H. Harada et al., "Research and development on heterogynous type and spectrum sharing type cognitive radio system," CROWNCOM, June 2009.

[5] IEEE Standard Coordinating Committee 41. http://www.scc41.org/

[6] IEEE Standard 1900.1-2008.

[7] IEEE Standard 1900.2-2008

[8] S. Buljore et al "Architecture and Enablers for Optimized Radio Resource Usage in Heterogeneous Wireless Access Networks: The IEEE 1900.4 Working Group," IEEE Communications Magazine, vol. 47, no. 1, pp. 122-129, Jan. 2009.

[9] K. Moessner et al, "Spectrum Sensing for Cognitive Radio Systems: Technical Aspects and Standardization Activities of IEEE 1900.6 Working Group," submitted to IEEE Wireless Communication Magazine Dec. 2009. 